\title{
Mortality in patients with diabetes mellitus and Addison's disease: a nationwide, matched, observational cohort study
}

\author{
Dimitrios Chantzichristos ${ }^{1,2}$, Anders Persson ${ }^{3}$, Björn Eliasson 1,2,3, Mervete Miftaraj3, \\ Stefan Franzén ${ }^{3}$, Ragnhildur Bergthorsdottir1,2, Soffia Gudbjörnsdottir 1,2,3, \\ Ann-Marie Svensson ${ }^{3}$ and Gudmundur Johannsson ${ }^{1,2}$ \\ ${ }^{1}$ Department of Internal Medicine and Clinical Nutrition, Institute of Medicine at Sahlgrenska Academy, \\ University of Gothenburg, Gothenburg, Sweden, ²Department of Endocrinology-Diabetes-Metabolism, \\ Sahlgrenska University Hospital, Gothenburg, Sweden, and ${ }^{3}$ Department of Medicine, National Diabetes Register, \\ Centre of Registers, University of Gothenburg, Gothenburg, Sweden
}

\author{
Correspondence \\ should be addressed \\ to D Chantzichristos \\ Email \\ dimitrios.chantzichristos@ \\ gu.se
}

\begin{abstract}
Objective: Our hypothesis was that patients with diabetes mellitus obtain an additional risk of death if they develop Addison's disease (AD).

Design and methods: Nationwide, matched, observational cohort study cross-referencing the Swedish National Diabetes Register with Inpatient, Cancer and Cause of Death Registers in patients with diabetes (type 1 and 2) and $A D$ and matched controls with diabetes. Clinical characteristics at baseline, overall, and cause-specific mortality were assessed. The relative risk of death was assessed using a Cox proportional hazards regression model.

Results: Between January 1996 and December 2012, 226 patients with diabetes and AD were identified and matched with 1129 controls with diabetes. Median (interquartile range) follow-up was 5.9 (2.7-8.6) years. When patients with diabetes were diagnosed with AD, they had an increased frequency of diabetes complications, but both medical history of cancer and coronary heart disease did not differ compared with controls. Sixty-four of the 226 patients with diabetes and AD (28\%) died, while 112 of the 1129 controls (10\%) died. The estimated relative risk increase (hazard ratio) in overall mortality in the diabetes and AD group was 3.89 (95\% confidence interval 2.84-5.32) compared with controls with diabetes. The most common cause of death was cardiovascular in both groups, but patients with diabetes and AD showed an increased death rate from diabetes complications, infectious diseases and unknown causes.

Conclusions: Patients with the rare combination of diabetes and AD showed a markedly increased mortality and died more frequently from infections and unknown causes than patients with diabetes alone. Improved strategy for the management of this combination of metabolic disorders is needed.

European Journal of Endocrinology (2017) 176, 31-39
\end{abstract}

\section{Introduction}

Diabetes mellitus (DM) type 1 and type 2 are established risk factors for cardiovascular disease (1) and strongly associated with increased risk of death (2). These associations are related to glycaemic control and the presence of co-morbidities such as hypertension (3) and DM-related nephropathy (4). For example, in patients with type 1 DM (T1DM), the risk of all-cause mortality is increased nearly 5 -fold in the presence of microalbuminuria (5).

Addison's disease (AD) or primary adrenal insufficiency is, in the Western world, most commonly due to autoimmune destruction of the adrenal cortex causing cortisol, aldosterone and androgen deficiency (6). Patients with AD may also have other autoimmune
() 2017 European Society of Endocrinology Printed in Great Britain
Published by Bioscientifica Ltd. 
manifestations, with approximately half of them having autoimmune thyroid disease and up to $14 \%$ also have T1DM (7). Patients with AD and either T1DM or autoimmune thyroid disease or both are classified as having autoimmune polyendocrine syndrome type 2 (8). Both T1DM and AD are deadly diseases unless treated with insulin and glucocorticoids respectively (9). Patients with $\mathrm{AD}$, receiving conventional replacement therapy, still have a 2-fold increased mortality risk compared with the general population $(10,11)$, which is mainly due to cancer, cardiovascular diseases and infections (10).

The combination of T1DM and $\mathrm{AD}$ is rare with an estimated prevalence of 20 cases per million inhabitants (7), which may explain the paucity of outcome data in this patient group. A small study of ten patients with T1DM and AD showed different basal and meal-related insulin requirements compared with patients having only T1DM (12). A recent study based on health insurance data showed increased risk of adrenal crisis among patients with T1DM and AD compared with patients with only AD (13), and an epidemiological study showed that patients with $\mathrm{AD}$ and $\mathrm{DM}$ had an increased risk of death compared with patients with only AD (10).

Having both DM (T1DM and type 2 DM (T2DM)) and $\mathrm{AD}$ is a complicated condition due to the complex metabolic interplay between insulin and cortisol. Our hypothesis is that mortality is increased in these patients above that seen among patients with DM alone. Using the detailed clinical information provided by the Swedish National Diabetes Register (NDR) combined with other Swedish national databases, we performed a nationwide, matched, observational study to determine the overall and cause-specific mortality in patients with DM from the time they were diagnosed with AD.

\section{Subjects and methods}

\section{Study design}

This is a nationwide, matched, observational cohort study using data from the Swedish NDR, the Swedish National Inpatient Register, the Swedish Cancer Register and the Swedish Cause of Death Register. The NDR is a quality register with a 97\% nationwide coverage (14) to which detailed clinical information on adult patients with DM is reported. Each patient provides informed consent for inclusion in the register. The Swedish National Inpatient Register includes data on all inpatient admissions in Sweden from 1987 onward. The Swedish Cancer Register includes data on cancer and the Swedish Cause of Death
Register includes data on causes of death. All registries are continuously validated, have a coverage of about 99\% and are linked to each other by a unique personal identification code $(15,16,17,18)$. The study was approved by the Regional Ethical Review Board in Gothenburg, Sweden.

\section{Procedures}

First, we identified patients with either T1DM or T2DM and $\mathrm{AD}$ in the Swedish National Inpatient Register between 1 January 1988 and 31 December 2012 (in a total population of 628475 individuals) (Fig. 1). Then, we crossmatched these patients with patients registered in the NDR having DM and AD. These patients with DM and AD in the NDR (cases) were, finally, matched to five controls with DM alone, also registered in NDR. The observation period was from 1 January 1996 (when the NDR started) to 31 December 2012. Patients were identified using the codes of the International Classification of Diseases, Ninth Revision (ICD-9) for years 1987-1996 and for ICD-10 for years 1997-2012. Codes from ICD-7 were used for cancer diagnosis in the Swedish Cancer Register. The codes used to identify DM were ICD-9 code 250 and ICD-10 codes E10-E14 and to identify patients with AD ICD-9 code 255.4 and ICD-10 codes E27.1 and/or E27.2. The search criteria for $\mathrm{AD}$ in the Swedish National Inpatient Register have been previously validated (10). Patients with endogenous Cushing's syndrome and/or any disorders of the pituitary gland were excluded. Details on all ICD-9 and ICD-10 codes used in this study are presented in Supplementary Table 1 , see section on supplementary data given at the

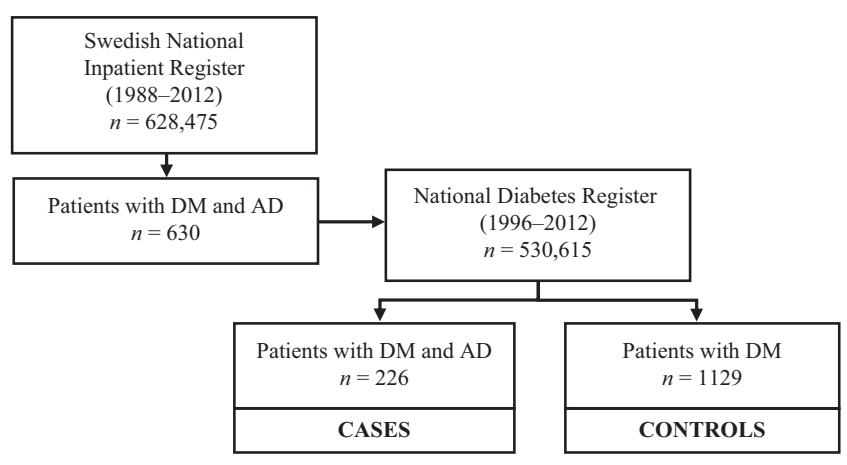

\section{Figure 1}

Study flow chart. Patients with diabetes mellitus (DM) and Addison's disease (AD) identified in the Swedish National Inpatient Register who were then identified in the Swedish National Diabetes Register (NDR) also were included in the primary analysis. For each case, five closely matched controls with only DM were identified in the NDR. 
end of this article. The period used to retrieve baseline data was between 1 year before and 2 weeks after the $\mathrm{AD}$ diagnosis for cases or inclusion in the study for controls.

Glycosylated haemoglobin concentrations (HbA1c) were analysed at local laboratories. Analyses of HbA1c are quality assured nationwide by regular calibration with the high-performance liquid chromatography Mono-S method, with measurements converted to $\mathrm{mmol} / \mathrm{mol}$ (19). We used the clinical classification of T1DM and T2DM, which is the diagnosis attributed to the patients by the physicians who reported them to the NDR.

\section{Outcomes}

After inclusion, all patients were monitored in the Swedish Cause of Death Register until they died or until end of cut-off on 31 December 2012. The primary outcomes were all-cause mortality and main cause of death, which is the primary cause of death as defined by a physician on the death certificate. Main causes of death, in accordance with previous studies in patients with $\mathrm{AD}$
$(11,20)$, were categorised as death from cardiovascular disease, cancer, DM complications, acute/chronic adrenal failure, infection (including sepsis), acute/chronic renal failure, gastrointestinal disease, chronic lung disease and sudden death.

\section{Statistical analysis}

Baseline was defined as the time when cases were diagnosed with both $\mathrm{DM}$ and $\mathrm{AD}$ in the NDR, and for matched controls, the time of NDR registration. Standardised mean difference (SMD) was used to estimate imbalances between the two groups with respect to the baseline variables. Imbalance was defined as an absolute SMD value $>20 \%$.

In the matching procedure, DM controls were matched 5:1, without replacement, to the cases based on the following variables: sex (male/female); DM type (1/2); year of birth (1902-1934, 1935-1943, 1944-1952 and 1953-); duration in years defined as the time from diagnosis of $\mathrm{DM}$ to $\mathrm{AD}$ diagnosis for cases and $\mathrm{DM}$

Table 1 Baseline demographics and measurements in patients with diabetes mellitus and Addison's disease and their matched controls with diabetes mellitus. Data are presented as $n$ (\%), mean (s.D.), or median (IQR) as appropriate for the whole study population (AII) and patients with T1DM and T2DM respectively. Standardised mean difference between the diabetes mellitus and Addison's disease (DM and AD) and DM control groups (shown in the last column when relevant) is a measure of size effect, with imbalance between the groups defined as an absolute value $>20 \%$.

\begin{tabular}{l}
\hline \\
\hline Age (year) \\
All \\
T1DM \\
T2DM \\
Female \\
All \\
T1DM \\
T2DM \\
DM \\
All \\
T1DM \\
T2DM \\
DM duration (year) \\
All \\
T1DM \\
T2DM \\
HbA1c (mmol/mol) \\
All \\
T1DM \\
T2DM \\
HbA1c (\%) \\
All \\
T1DM \\
T2DM \\
\hline
\end{tabular}

\begin{tabular}{c} 
All \\
$(n=1355)$ \\
\hline $53.8(19.1)$ \\
$45.5(16.5)$ \\
$69.7(12.7)$ \\
\\
$636(47 \%)$ \\
$426(48 \%)$ \\
$210(45 \%)$ \\
$1355(100 \%)$ \\
$887(65 \%)$ \\
$468(35 \%)$ \\
$7.0(2.0-14.0)$ \\
$10.0(3.0-18.0)$ \\
$3.0(1.0-8.0)$ \\
$60(15)$ \\
$63(15)$ \\
$53(12)$ \\
$7.6(1.4)$ \\
$7.9(1.4)$ \\
$7.0(1.1)$ \\
\end{tabular}

\begin{tabular}{c}
$\begin{array}{c}\text { Diabetes mellitus and } \\
\text { Addison's disease }(n=226)\end{array}$ \\
\hline $52.3(20.1)$ \\
$43.2(17.3)$ \\
$69.5(12.4)$ \\
$106(47 \%)$ \\
$71(48 \%)$ \\
$35(45 \%)$ \\
$226(100 \%)$ \\
$148(65 \%)$ \\
$78(35 \%)$ \\
$6.7(0.5-13.7)$ \\
$9.9(3.0-16.1)$ \\
$2.1(0.2-7.0)$ \\
$62(15)$ \\
$64(14)$ \\
$58(15)$ \\
$7.8(1.3)$ \\
$8.0(1.3)$ \\
$7.5(1.3)$ \\
\end{tabular}

\begin{tabular}{c}
$\begin{array}{c}\text { Diabetes mellitus controls } \\
(n=1129)\end{array}$ \\
\hline $54.1(18.9)$ \\
$45.9(16.3)$ \\
$69.7(12.8)$ \\
\\
$530(47 \%)$ \\
$355(48 \%)$ \\
$175(45 \%)$ \\
$1129(100 \%)$ \\
$739(65 \%)$ \\
$390(35 \%)$ \\
$7.0(3.0-14.0)$ \\
$10.0(3.0-19.0)$ \\
$3.0(1.0-8.0)$ \\
$60(15)$ \\
$63(15)$ \\
$53(12)$ \\
$7.6(1.4)$ \\
$7.9(1.4)$ \\
$7.0(1.1)$ \\
\end{tabular}

\begin{tabular}{c}
$\begin{array}{c}\text { Standardised mean } \\
\text { difference (\%) }\end{array}$ \\
\hline 9.3 \\
15.9 \\
1.2 \\
- \\
- \\
- \\
- \\
- \\
- \\
- \\
- \\
- \\
-16.8 \\
-6.6 \\
-42.7 \\
-16.8 \\
-6.6 \\
-42.7 \\
\end{tabular}

IQR, interquartile range; s.D., standard deviation; T1DM, type 1 diabetes mellitus; T2DM, type 2 diabetes mellitus. 
duration until inclusion for controls (categorised as $0-3$, $3-8,8-14,14-)$; and calendar year of DM and AD diagnosis for cases and year of registration in the NDR for controls (1996-2006, 2007-2009, 2010-2011, 2012).

A Cox proportional hazards model was used to evaluate the mortality of cases compared with matched controls. Factors included in the model were year of DM diagnosis, year of inclusion in the study, the duration between these two dates, sex and the two groups of patients (DM and AD vs DM). Type of DM was used as a stratification factor due to the different shape of the hazard functions for patients with T1DM and T2DM

Table 2 Medical history and co-morbidity in the 226 patients with diabetes mellitus and Addison's disease and their 1129 matched controls with diabetes mellitus. Data are presented as $n$ (\%) for the whole study population (All) and patients with T1DM and T2DM respectively. Standardised mean difference between the diabetes mellitus and Addison's disease (DM and AD) and DM control groups is a measure of size effect, with imbalance between the groups defined as an absolute value $>20 \%$.

\begin{tabular}{|c|c|c|c|c|}
\hline & $\begin{array}{c}\text { All } \\
(n=1355)\end{array}$ & $\begin{array}{c}\text { Diabetes mellitus and } \\
\text { Addison's disease }(n=226)\end{array}$ & $\begin{array}{l}\text { Diabetes mellitus controls } \\
\qquad(n=1129)\end{array}$ & $\begin{array}{l}\text { Standardised mean } \\
\text { difference }(\%)\end{array}$ \\
\hline \multicolumn{5}{|c|}{ Diabetic nephropathy } \\
\hline All & $29(2.1 \%)$ & $11(4.9 \%)$ & $18(1.6 \%)$ & -16.61 \\
\hline T1DM & $23(2.6 \%)$ & $8(5.4 \%)$ & $15(2.0 \%)$ & \\
\hline T2DM & $6(1.3 \%)$ & $3(3.8 \%)$ & $3(0.8 \%)$ & \\
\hline \multicolumn{5}{|c|}{ Diabetic retinopathy } \\
\hline All & $91(6.7 \%)$ & $36(16.0 \%)$ & $55(4.9 \%)$ & -32.85 \\
\hline T1DM & $83(9.4 \%)$ & $31(21.0 \%)$ & $52(7.0 \%)$ & \\
\hline T2DM & $8(1.7 \%)$ & $5(6.4 \%)$ & $3(0.8 \%)$ & \\
\hline \multicolumn{5}{|c|}{ Diabetic neuropathy } \\
\hline All & $41(3.0 \%)$ & $16(7.1 \%)$ & $25(2.2 \%)$ & -20.83 \\
\hline T1DM & $30(3.4 \%)$ & $11(7.4 \%)$ & $19(2.6 \%)$ & \\
\hline T2DM & $11(2.4 \%)$ & $5(6.4 \%)$ & $6(1.5 \%)$ & \\
\hline \multicolumn{5}{|c|}{ Diabetic angiopathy } \\
\hline All & $42(3.1 \%)$ & $15(6.6 \%)$ & $27(2.4 \%)$ & -18.22 \\
\hline T1DM & $19(2.1 \%)$ & $8(5.4 \%)$ & $11(1.5 \%)$ & \\
\hline T2DM & $23(4.9 \%)$ & $7(9.0 \%)$ & $16(4.1 \%)$ & \\
\hline \multicolumn{5}{|c|}{ Multiple diabetic complications } \\
\hline All & $62(4.6 \%)$ & $26(11.0 \%)$ & $36(3.2 \%)$ & -29.08 \\
\hline T1DM & $54(6.1 \%)$ & $21(14.0 \%)$ & $33(4.5 \%)$ & \\
\hline T2DM & $8(1.7 \%)$ & $5(6.4 \%)$ & $3(0.8 \%)$ & \\
\hline \multicolumn{5}{|c|}{ Hypertension } \\
\hline All & $236(17.0 \%)$ & $43(19.0 \%)$ & $193(17 \%)$ & -4.13 \\
\hline T1DM & $84(9.5 \%)$ & $9(6.1 \%)$ & $75(10 \%)$ & \\
\hline T2DM & $152(32.0 \%)$ & $34(44.0 \%)$ & $118(30 \%)$ & \\
\hline \multicolumn{5}{|c|}{ Coronary heart disease including acute myocardial infarction } \\
\hline All & $151(11.0 \%)$ & $36(16.0 \%)$ & $115(10.0 \%)$ & -14.43 \\
\hline T1DM & $47(5.3 \%)$ & $11(7.4 \%)$ & $36(4.9 \%)$ & \\
\hline T2DM & $104(22.2 \%)$ & $25(32.1 \%)$ & $79(20.3 \%)$ & \\
\hline \multicolumn{5}{|c|}{ Congestive heart failure } \\
\hline All & $66(4.9 \%)$ & $18(8.0 \%)$ & $48(4.3 \%)$ & -13.35 \\
\hline T1DM & $12(1.4 \%)$ & $2(1.4 \%)$ & $10(1.4 \%)$ & \\
\hline T2DM & $54(11.0 \%)$ & $16(20.0 \%)$ & $38(9.7 \%)$ & \\
\hline \multicolumn{5}{|l|}{ Stroke } \\
\hline All & $57(4.2 \%)$ & $8(3.5 \%)$ & $49(4.3 \%)$ & 3.31 \\
\hline T1DM & $14(1.6 \%)$ & $3(2.0 \%)$ & $11(1.5 \%)$ & \\
\hline T2DM & $43(9.2 \%)$ & $5(6.4 \%)$ & $38(9.7 \%)$ & \\
\hline \multicolumn{5}{|c|}{ Infections requiring hospital admission } \\
\hline All & $223(16 \%)$ & $78(34 \%)$ & $145(13 \%)$ & -45.69 \\
\hline T1DM & $138(16 \%)$ & $46(31 \%)$ & $92(12 \%)$ & \\
\hline T2DM & $85(18 \%)$ & $32(41 \%)$ & $53(14 \%)$ & \\
\hline \multicolumn{5}{|l|}{ Cancer } \\
\hline All & $93(6.9 \%)$ & $16(7.1 \%)$ & $77(6.8 \%)$ & -0.84 \\
\hline T1DM & $27(3.0 \%)$ & $2(1.4 \%)$ & $25(3.4 \%)$ & \\
\hline T2DM & $66(14.0 \%)$ & $14(18.0 \%)$ & $52(13.0 \%)$ & \\
\hline
\end{tabular}

T1DM, type 1 diabetes mellitus; T2DM, type 2 diabetes mellitus. 
respectively. The relative risk of mortality of $\mathrm{DM}$ and $\mathrm{AD}$ vs $\mathrm{DM}$, as well as T1DM and $\mathrm{AD}$ vs T1DM and T2DM and $\mathrm{AD}$ vs $\mathrm{T} 2 \mathrm{DM}$, were estimated by hazard ratios (HR) and their corresponding 95\% confidence intervals (CIs). Survival curves were determined for matched data using the Kaplan-Meier method.

\section{Results}

\section{Baseline}

We identified 630 patients with DM who then developed $\mathrm{AD}$ or were registered with both diagnoses at the same time in the Swedish National Inpatient Register between 1 January 1988 and 31 December 2012 (Fig. 1). Among these, 296 patients were registered after 1 January 1996, and we studied 226 of them who were also registered in the NDR. The 226 patients with DM and AD were matched to 1129 patients with DM in the NDR dataset containing 530615 eligible patients. The median follow-up time of the patients was 5.9 years (IQR: 2.7-8.6), and the total study follow-up time was 8290 patient-years.

For both groups combined $(n=1355), 887$ patients (65\%) had T1DM and 636 (47\%) were female (Table 1). Patients with T1DM were diagnosed with $\mathrm{AD}$ at a mean age of 43.2 years (s.D. 17.3) after a median DM duration of 9.9 years (IQR: 3-16.1), whereas T2DM patients were diagnosed at a mean age of 69.5 years (s.D. 12.4) after a median DM duration of 2.1 years (IQR: 0.2-7).

At baseline, $\mathrm{HbA} 1 \mathrm{c}$ in cases was $62.1 \mathrm{mmol} / \mathrm{mol}$ (S.D. 14.7 ) or $7.8 \%$ (S.D. 1.3) and in controls $59.6 \mathrm{mmol} / \mathrm{mol}$ (s.D. 14.7) or $7.6 \%$ (s.D. 1.4) with a SMD of 16.6 (Table 1). The proportion of DM complications at baseline (retinopathy, neuropathy and multiple DM complications) was higher in cases than that in controls. In contrast, the proportion of DM nephropathy and angiopathy did not differ among the two groups (Table 2). The two patient groups did not differ in terms of medical history of hypertension, coronary heart disease including acute myocardial infarction, congestive heart failure, stroke and cancer. Medical history of infections requiring inpatient admission to hospital was increased in the $\mathrm{DM}$ and $\mathrm{AD}$ group compared with the DM controls (SMD =45.7).

\section{Mortality}

The observed number of deaths was 64 in the 226 cases $(28 \%)$ and 112 deaths in the 1129 controls (10\%) (Table 3). The adjusted relative risk increase (HR) in overall mortality of the $\mathrm{DM}$ and $\mathrm{AD}$ group was therefore 3.89 (95\% CI: 2.84-5.32) compared with the DM controls (Fig. 2). The adjusted relative risk increase in overall mortality was 4.28 (95\% CI: 2.59-6.95) for patients with T1DM and AD, and 3.81 (95\% CI: 2.52-5.75) for those with T2DM and AD compared with controls.

\section{Cause-specific mortality}

There were differences in the main causes of death between patients with $\mathrm{DM}$ and $\mathrm{AD}$ and patients with only DM (Fig. 3). The most common main cause of death was cardiovascular disease in both groups, whereas deaths from DM complications, infections and unknown causes were more frequent among patients with $\mathrm{DM}$ and $\mathrm{AD}$. Death from cancer was more frequent among patients with only DM.

\section{Independent analysis of the Swedish National Inpatient Register cohort}

In a separate mortality analysis, we matched the 630 patients with both DM and AD identified in the Swedish National Inpatient Register with 3138 controls with DM in NDR. Mean (s.D.) age at baseline was 57.2 (19.3) years for the cases and 58.8 (16.9) years for the controls. Median (IQR) DM duration was $1.2(0-6)$ and $4(2-7)$ years for cases and controls respectively. In the group of patients with $\mathrm{DM}$ and $\mathrm{AD}, 53.7 \%$ were female and $37.8 \%$ had T1DM, and in controls, $53.6 \%$ were female and $37.9 \%$ had T1DM.

Table 3 Deaths in patients with DM and AD and their matched controls with DM identified in the Swedish National Diabetes Register and from the larger cohort of such patients identified in the Swedish National Inpatient Register.

\begin{tabular}{|c|c|c|c|c|c|}
\hline & \multicolumn{5}{|c|}{ No. of patients } \\
\hline & All & T1DM & T2DM & Females & Males \\
\hline NDR patients with DM and AD $(n=226)$ & $64(28 \%)$ & $29(20 \%)$ & $35(45 \%)$ & $33(31 \%)$ & $31(26 \%)$ \\
\hline NDR controls with DM $(n=1129)$ & $112(9.9 \%)$ & $35(4.7 \%)$ & $77(20 \%)$ & $54(10 \%)$ & $58(9.7 \%)$ \\
\hline SNIR patients with DM and AD $(n=630)$ & $386(61 \%)$ & $62(26 \%)$ & $324(83 \%)$ & $221(65 \%)$ & $165(56 \%)$ \\
\hline
\end{tabular}

NDR, National Diabetes Register; SNIR, Swedish National Inpatient Register; T1DM, type 1 diabetes mellitus; T2DM, type 2 diabetes mellitus. 


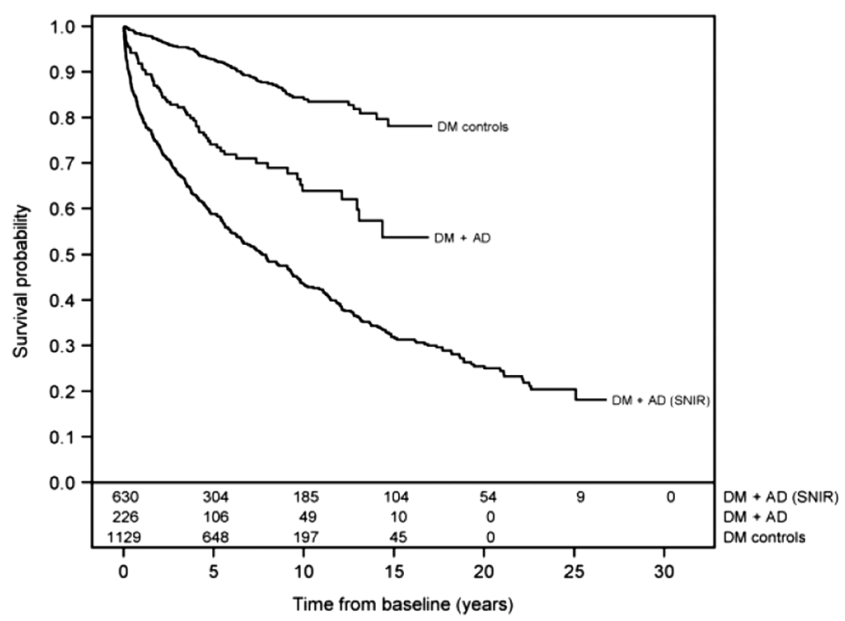

Figure 2

Cumulative overall mortality in patients with diabetes mellitus and Addison's disease (DM+AD) in the Swedish National Diabetes Register vs their matched controls with diabetes mellitus (DM) in the Swedish National Diabetes Register vs patients with diabetes mellitus and Addison's disease (DM+AD) in the Swedish National Inpatient Register. The adjusted relative risk increase (hazard ratio) in overall mortality in cases from the Swedish National Diabetes Register was 3.89 (95\% Cl: 2.84-5.32) compared with the controls (the duration of observation was 17 years). The adjusted relative risk increase (hazard ratio) in overall mortality in cases from the Swedish National Inpatient Register was 6.62 (95\% Cl: 5.80-7.55) compared with the controls (the duration of observation was 27 years).

The observed number of deaths was 386 in the 630 cases $(61 \%)$ (Table 3$)$, and the adjusted relative risk increase in overall mortality in these patients

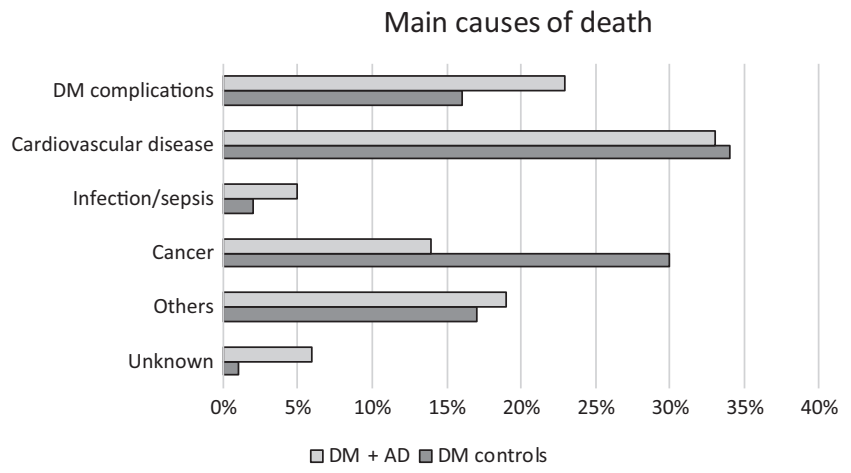

Figure 3

Main disease-specific causes of death in patients with diabetes mellitus and Addison's (DM + AD, $n=226)$ and matched controls with diabetes mellitus (DM, $n=1129$ ). was 6.62 (95\% CI: 5.80-7.55) compared with their controls (Fig. 2).

\section{Discussion}

This nationwide study of patients with DM and AD in Sweden showed a near 4-fold increased risk of all-cause mortality compared with matched controls with DM. The most common cause of death in patients with DM and $\mathrm{AD}$ was cardiovascular disease, but death due to DM complications, infections or unknown causes was more common than that in patients with DM alone. At inclusion, degree of co-morbidities and glycaemic control did not differ markedly between patients with DM and $\mathrm{AD}$ and their controls, suggesting an impact of $\mathrm{AD}$ on the excess mortality.

Patients with DM have an increased mortality rate. Higher HbA1c in both T1DM and T2DM patients, and young age in T2DM patients are related to higher mortality $(5,21)$. In addition, nephropathy is a serious co-morbidity increasing the risk of death in patients with $\mathrm{DM}(5,21)$. The most common diabetes complications in T1DM patients are microvascular $(5,22)$, especially in those with long DM duration, whereas in T2DM patients, the most common diabetes complications are macrovascular (21). In this study, we demonstrated that $\mathrm{AD}$, as another DM co-morbidity, increased the overall risk of death in patients with DM.

A possible explanation for these deleterious effects of $\mathrm{AD}$ on mortality rate in patients with $\mathrm{DM}$ is the counter-balancing metabolic effects of insulin and cortisol, the main treatments for $\mathrm{DM}$ and $\mathrm{AD}$ respectively. Although treatment for DM addresses insulin resistance, enhancement of insulin secretion, or insulin replacement, treatment of $\mathrm{AD}$ means replacement therapy with glucocorticoids (mainly hydrocortisone). Glucocorticoids, as the name implies, stimulate glycogen deposition and increase hepatic glucose output and inhibit glucose uptake and utilisation in peripheral tissues, leading to insulin resistance and increased plasma glucose levels $(23,24)$. This is why patients with T1DM present with reduced insulin requirement when they develop severe glucocorticoid deficiency due to AD (25) and why the risk of T2DM is increased in patients treated with pharmacological doses of glucocorticoids (26). The doses of glucocorticoids used for replacement should be sufficient to prevent adrenal crisis but not too high to induce side effects, such as obesity, hypertension and DM $(27,28,29)$. Also, in patients with insulin-treated DM, the 
timing of the hydrocortisone dose in relation to the intake of meals and administration of insulin is of importance due to the short half-life of hydrocortisone.

The main cause of death was cardiovascular diseases in both patients with DM with and without AD. Patients with $\mathrm{DM}$ and $\mathrm{AD}$, however, died more frequently from complications related to DM, infections (including sepsis) and unknown causes. The increased rate of death from DM-related complications might be due to the higher proportion of almost all DM complications at baseline in patients with $\mathrm{DM}$ and $\mathrm{AD}$. The explanation for this is unclear, but may be related to the fact that management of DM may be more difficult if the intrinsic regulation of cortisol secretion is impaired, which is likely to occur well in advance of the actual diagnosis of AD. Another explanation might be that patients with uncomplicated DM are treated by general practitioners, whereas DM patients with complications and $\mathrm{AD}$ are referred to specialists.

Patients with autoimmune diseases have an increased risk of cancer (30) and two previous Swedish studies in patients with $\mathrm{AD}$ and patients with autoimmune polyendocrine syndrome type 2 have shown increased death from cancer $(10,11)$. Our data show reduced cancer mortality (as the main cause of death) in patients with $\mathrm{DM}$ and $\mathrm{AD}$ compared with patients with $\mathrm{DM}$ suggesting that $\mathrm{AD}$ or its treatment with glucocorticoids may modify the increased risk of cancer death among patients with DM.

Patients with $\mathrm{AD}$ are at risk of developing adrenal crisis, a life-threatening condition, if not rapidly treated with parenteral glucocorticoids and saline infusion. Therefore, patients with $\mathrm{AD}$ are taught to increase the dose of glucocorticoids in the event of infections or other physical and mental stress events to prevent a crisis. Previous studies have shown increased risk of adrenal crisis among patients with both T1DM and $\mathrm{AD}(13,20$, 31). This study supports these previous observations by showing higher mortality from infections and unknown cause that may be explained by untreated or inadequately treated adrenal crisis, severe hypoglycaemia or their combination. These data indicate that patients with DM and AD need targeted education for managing both their $\mathrm{DM}$ and $\mathrm{AD}$ during an infection or other stressful events.

Data from the larger cohort generated from the Swedish National Inpatient Register were analysed as an independent study of the more selected cohort from the NDR. This analysis confirmed our primary analysis of excess mortality among patients with DM and AD and also suggested that the primary analysis did not overestimate the risk of death. The Kaplan-Meier probability curve in patients with $\mathrm{DM}$ and $\mathrm{AD}$ in the Inpatient Register had a steeper inclination during the first few years after $\mathrm{AD}$ was diagnosed in contrast to the probability curve for patients with $\mathrm{DM}$ and $\mathrm{AD}$ in our primary cohort. This is in agreement with previous findings showing that $\mathrm{AD}$ patients are at increased risk of death close to the time of diagnosis (10).

This study has several strengths. First, a detailed description of the patients at baseline was possible from the NDR, which has a high nationwide coverage in Sweden. Second, our search criteria for AD in the Swedish National Inpatient Register have been validated in a previous study (10). Finally, most of the reported main causes of death have been previously validated and shown to have a high reliability (18).

One of the limitations of this study is the missing data around the period of the AD diagnosis in the NDR that made us define baseline as the data registered 1 year before the diagnosis and up to 2 weeks after the diagnosis. However, support for this decision is that $\mathrm{AD}$ emerges by a gradual destruction of the adrenal cortex before overt cortisol deficiency develops. Another limitation is that the registries used in this study have no information on patients' glucocorticoid replacement regimen.

In conclusion, this nationwide study shows that DM patients with $\mathrm{AD}$ have a substantially increased risk of death compared with closely matched patients with DM. The disease-specific mortality in patients with both $\mathrm{DM}$ and $\mathrm{AD}$ is increased for infections and unknown causes, suggesting that death due to adrenal crisis is a contributing factor. As adrenal crisis can be prevented by an adequate rescue regimen, this study highly alerts the risk of premature death from infections and other stressrelated events when patients with both $\mathrm{DM}$ and $\mathrm{AD}$ are not treated properly. Better patient education as well as a combined effort between physicians treating DM and those treating $\mathrm{AD}$ should be directed at improving the care of this patient group with a complex metabolic disease who, otherwise, have a very poor outcome as shown in this study.

Supplementary data

This is linked to the online version of the paper at http://dx.doi.org/10.1530/ EJE-16-0657.

Declaration of interest

The authors declare that there is no conflict of interest that could be perceived as prejudicing the impartiality of the research reported. 


\section{Funding}

The National Diabetes Registry (NDR) receives financial support from the Swedish Association of Local Authorities and Regions and the Region Västra Götaland. The study was supported in part by the Swedish government under an ALF agreement (G J).

The funders of the study had no role in study design, data collection, data analysis, data interpretation or writing of the report. All authors had full access to all data in the study and shared the final responsibility for the decision to submit for publication.

\section{Author contribution statement}

All authors contributed to the design of the study and interpretation of the data. A P, M M and A-M S acquired data. A P, D C, M M, S F and A-M S performed the statistical analyses. D C drafted the report. All authors contributed to the writing and to the final version of the manuscript.

\section{Acknowledgements}

The authors thank Peter Todd (Tajut Ltd., Kaiapoi, New Zealand) for third-party writing assistance in drafting of this manuscript, for which he received financial compensation from ALF funding.

A part of the study results has previously been presented as an oral presentation at ENDO 2016 in Boston, USA.

\section{References}

1 Sarwar N, Gao P, Seshasai SR, Gobin R, Kaptoge S, Di Angelantonio E, Ingelsson E, Lawlor DA, Selvin E, Stampfer M et al. Diabetes mellitus, fasting blood glucose concentration, and risk of vascular disease: a collaborative meta-analysis of 102 prospective studies. Lancet 2010375 2215-2222. (doi:10.1016/ S0140-6736(10)60484-9)

2 Seshasai SR, Kaptoge S, Thompson A, Di Angelantonio E, Gao P, Sarwar N, Whincup PH, Mukamal KJ, Gillum RF, Holme I et al. Diabetes mellitus, fasting glucose, and risk of cause-specific death. New England Journal of Medicine 2011364 829-841. (doi:10.1056/ NEJMoa1008862)

3 American Diabetes Association. Standards of medical care in diabetes - 2014. Diabetes Care 201437 (Supplement 1) S14-S80. (doi:10.2337/ dc14-S014)

4 Groop PH, Thomas MC, Moran JL, Waden J, Thorn LM, Makinen VP, Rosengard-Barlund M, Saraheimo M, Hietala K, Heikkila O et al. The presence and severity of chronic kidney disease predicts allcause mortality in type 1 diabetes. Diabetes 200958 1651-1658. (doi:10.2337/db08-1543)

5 Lind M, Svensson A-M, Kosiborod M, Gudbjörnsdottir S, Pivodic A, Wedel H, Dahlqvist S, Clements M \& Rosengren A. Glycemic control and excess mortality in type 1 diabetes. New England Journal of Medicine 2014371 1972-1982. (doi:10.1056/NEJMoa1408214)

6 Lovas K \& Husebye ES. Addison's disease. Lancet 2005365 2058-2061. (doi:10.1016/S0140-6736(05)66700-1)

7 Lovas K \& Husebye ES. High prevalence and increasing incidence of Addison's disease in western Norway. Clinical Endocrinology 200256 787-791. (doi:10.1046/j.1365-2265.2002.t01-1-01552.x)

8 Betterle C, Dal Pra C, Mantero F \& Zanchetta R. Autoimmune adrenal insufficiency and autoimmune polyendocrine syndromes: autoantibodies, autoantigens, and their applicability in diagnosis and disease prediction. Endocrine Reviews 200223 327-364. (doi:10.1210/ edrv.23.3.0466)

9 Dunlop D. Eighty-six cases of addison's disease. BMJ 19632 887-891. (doi:10.1136/bmj.2.5362.887)
10 Bergthorsdottir R, Leonsson-Zachrisson M, Oden A \& Johannsson G. Premature mortality in patients with Addison's disease: a populationbased study. Journal of Clinical Endocrinology and Metabolism 200691 4849-4853. (doi:10.1210/jc.2006-0076)

11 Bensing S, Brandt L, Tabaroj F, Sjoberg O, Nilsson B, Ekbom A, Blomqvist P \& Kampe O. Increased death risk and altered cancer incidence pattern in patients with isolated or combined autoimmune primary adrenocortical insufficiency. Clinical Endocrinology 200869 697-704. (doi:10.1111/j.1365-2265.2008.03340.x)

12 Elbelt U, Hahner S \& Allolio B. Altered insulin requirement in patients with type 1 diabetes and primary adrenal insufficiency receiving standard glucocorticoid replacement therapy. European Journal of Endocrinology 2009160 919-924. (doi:10.1530/EJE-08-1003)

13 Meyer G, Badenhoop K \& Linder R. Addison's disease with polyglandular autoimmunity carries a more than 2.5 -fold risk for adrenal crises: German Health insurance data 2010-2013. Clinical Endocrinology 201685 347-353. (doi:10.1111/cen.13043)

14 Rawshani A, Landin-Olsson M, Svensson AM, Nystrom L, Arnqvist HJ, Bolinder J \& Gudbjornsdottir S. The incidence of diabetes among 0-34 year olds in Sweden: new data and better methods. Diabetologia 201457 1375-1381. (doi:10.1007/s00125-014-3225-9)

15 Eliasson B \& Gudbjornsdottir S. Diabetes care - improvement through measurement. Diabetes Research and Clinical Practice 2014106 (Supplement 2) S291-S294. (doi:10.1016/S0168-8227(14)70732-6)

16 Ludvigsson JF, Andersson E, Ekbom A, Feychting M, Kim JL, Reuterwall C, Heurgren M \& Olausson PO. External review and validation of the Swedish national inpatient register. BMC Public Health 201111 450. (doi:10.1186/1471-2458-11-450)

17 Barlow L, Westergren K, Holmberg L \& Talback M. The completeness of the Swedish Cancer Register: a sample survey for year 1998. Acta Oncologica 200948 27-33. (doi:10.1080/02841860802247664)

18 Johansson LA, Bjorkenstam C \& Westerling R. Unexplained differences between hospital and mortality data indicated mistakes in death certification: an investigation of 1094 deaths in Sweden during 1995. Journal of Clinical Epidemiology 200962 1202-1209. (doi:10.1016/j.jclinepi.2009.01.010)

19 Geistanger A, Arends S, Berding C, Hoshino T, Jeppsson JO, Little R, Siebelder C \& Weykamp C. Statistical methods for monitoring the relationship between the IFCC reference measurement procedure for hemoglobin A1c and the designated comparison methods in the United States, Japan, and Sweden. Clinical Chemistry 200854 1379-1385. (doi:10.1373/clinchem.2008.103556)

20 Erichsen MM, Lovas K, Fougner KJ, Svartberg J, Hauge ER, Bollerslev J, Berg JP, Mella B \& Husebye ES. Normal overall mortality rate in Addison's disease, but young patients are at risk of premature death. European Journal of Endocrinology 2009160 233-237. (doi:10.1530/EJE08-0550)

21 Tancredi M, Rosengren A, Svensson A-M, Kosiborod M, Pivodic A, Gudbjörnsdottir S, Wedel H, Clements M, Dahlqvist S \& Lind M. Excess mortality among persons with type 2 diabetes. New England Journal of Medicine 2015373 1720-1732. (doi:10.1056/ NEJMoa1504347)

22 Nathan DM. The diabetes control and complications trial/ epidemiology of diabetes interventions and complications study at 30 years: overview. Diabetes Care 201437 9-16. (doi:10.2337/ dc13-2112)

23 Arnaldi G, Angeli A, Atkinson AB, Bertagna X, Cavagnini F, Chrousos GP, Fava GA, Findling JW, Gaillard RC, Grossman AB et al. Diagnosis and complications of Cushing's syndrome: a consensus statement. Journal of Clinical Endocrinology and Metabolism $2003 \mathbf{8 8}$ 5593-5602. (doi:10.1210/jc.2003-030871)

24 van Raalte DH, Ouwens DM \& Diamant M. Novel insights into glucocorticoid-mediated diabetogenic effects: towards expansion of therapeutic options? European Journal of Clinical Investigation 200939 81-93. (doi:10.1111/j.1365-2362.2008.02067.x) 
25 Armstrong L \& Bell PM. Addison's disease presenting as reduced insulin requirement in insulin dependent diabetes. BMJ $1996 \mathbf{3 1 2}$ 1601-1602. (doi:10.1136/bmj.312.7046.1601)

26 Gurwitz JH, Bohn RL, Glynn RJ, Monane M, Mogun H \& Avorn J. Glucocorticoids and the risk for initiation of hypoglycemic therapy. Archives of Internal Medicine 1994 154 97-101. (doi:10.1001/archi nte.1994.00420010131015)

27 Johannsson G, Falorni A, Skrtic S, Lennernas H, Quinkler M, Monson JP \& Stewart PM. Adrenal insufficiency: review of clinical outcomes with current glucocorticoid replacement therapy. Clinical Endocrinology 201582 2-11. (doi:10.1111/cen.12603)

28 Filipsson H, Monson JP, Koltowska-Haggstrom M, Mattsson A \& Johannsson G. The impact of glucocorticoid replacement regimens on metabolic outcome and comorbidity in hypopituitary patients. Journal of Clinical Endocrinology and Metabolism 200691 3954-3961. (doi:10.1210/jc.2006-0524)
29 Christiansen JJ, Djurhuus CB, Gravholt CH, Iversen P, Christiansen JS, Schmitz O, Weeke J, Jorgensen JO \& Moller N. Effects of cortisol on carbohydrate, lipid, and protein metabolism: studies of acute cortisol withdrawal in adrenocortical failure. Journal of Clinical Endocrinology and Metabolism 200792 3553-3559. (doi:10.1210/jc.2007-0445)

30 Zendehdel K, Nyren O, Ostenson CG, Adami HO, Ekbom A \& Ye W. Cancer incidence in patients with type 1 diabetes mellitus: a population-based cohort study in Sweden. Journal of the National Cancer Institute 200395 1797-1800. (doi:10.1093/jnci/djg105)

31 Hahner S, Spinnler C, Fassnacht M, Burger-Stritt S, Lang K, Milovanovic D, Beuschlein F, Willenberg HS, Quinkler M \& Allolio B. High incidence of adrenal crisis in educated patients with chronic adrenal insufficiency: a prospective study. Journal of Clinical Endocrinology and Metabolism 2015100 407-416. (doi:10.1210/ jc.2014-3191)

Received 3 August 2016

Revised version received 5 October 2016

Accepted 12 October 2016 